\title{
DYNAMICS OF A FREE PITCHING FLEXIBLE CANTILEVER NACA0012 AIRFOIL AT TRANSITIONAL REYNOLDS NUMBERS
}

\author{
Crystal Itwar Barrett \\ Department of Mechanical and \\ Aerospace Engineering \\ Royal Military College of Canada \\ Kingston, Ontario, Canada \\ Crystal.Itwar-Barrett@rmc.ca
}

\author{
Dr. Dominique Poirel \\ Department of Mechanical and \\ Aerospace Engineering \\ Royal Military College of Canada \\ Kingston, Ontario, Canada \\ poirel-d@rmc.ca
}

\author{
Dr. Asad Asghar \\ Department of Mechanical and \\ Aerospace Engineering \\ Royal Military College of Canada \\ Kingston, Ontario, Canada \\ Asad.Asghar@rmc.ca
}

\begin{abstract}
The dynamics of a free pitching flexible cantilever NACA 0012 airfoil were investigated at transitional Reynolds numbers. This work builds on previous investigations based on a quasi-2D rigid wing, moving elastically in pitch and heave. Wind tunnel tests were performed at various speeds, and three limit cycle oscillation (LCO) branches were observed. Further work is required to supplement this preliminary analysis, such as modeling, FEA simulation, and evaluation of the strain and acceleration information of the wing deformation
\end{abstract}

Keywords-transitional Reynolds numbers, flexible wing, LCO, free pitching, NACA0012, flexible cantilever

\section{INTRODUCTION}

The purpose of this series of experiments is to characterize the dynamics of a free pitching flexible cantilever NACA0012 wing at transitional Reynolds numbers. The wing experiences both rigid body and flexible body mode dynamics. It is well noted that the aerodynamics at transitional Reynolds numbers are complex, and include such phenomena as laminar boundary layer separation, which subsequently forms a laminar separation bubble (LSB) [1]. This paper is an extension of previous work performed on a rigid, elastically mounted in pitch and heave, quasi-2D NACA0012 wing.

\section{A. Wind Tunnel}

The tests were performed using the RMC wind tunnel, which is a closed circuit low speed tunnel powered by a $75 \mathrm{~kW}$ threephase motor. The flow velocity is controlled by varying the fan speed. The freestream velocity was measured with a pitot static tube (located approximately 9 chords upstream of airfoil), which was connected to an analog pressure transducer. The test section inner dimensions are $1.07 \mathrm{~m} \mathrm{x} 0.76 \mathrm{~m}$. The turbulence intensity of the tunnel is below $0.2 \%$ [1]. There is also a safety net located approximately 3 chords downstream from the wing.

\section{B. Flexible Wing Configuration}

The wing testing apparatus consists of the flexible NACA 0012 wing, mounted over a thin cantilever steel beam. Its kinematics can be seen in Fig. 1. The beam is fixed at its root to a free rotary base. The beam provides the structural stiffness, while the wing provides the aerodynamics loads. The wing and beam parameters can be seen in Tables I and II, respectively. The mass moments of inertia of the wing, beam and base can be seen in Table III.

TABLE I. WING PARAMETERS

\begin{tabular}{|c|c|}
\hline Material & Fiberglass epoxy, foam, and plastic \\
\hline Span & $0.445 \mathrm{~m}$ \\
\hline Chord & $0.15 \mathrm{~m}$ \\
\hline Aspect Ratio & 3 \\
\hline Mass & $0.145 \mathrm{~kg}$ \\
\hline
\end{tabular}

TABLE II. BEAM PARAMETERS

\begin{tabular}{|c|c|}
\hline Material & AISI O1 steel \\
\hline Length (exposed) & $0.45 \mathrm{~m}$ \\
\hline Width & $0.051 \mathrm{~m}$ \\
\hline Thickness & $0.0014 \mathrm{~m}$ \\
\hline Young's Modulus & $200 \mathrm{GPa}$ \\
\hline Mass & $0.222 \mathrm{~kg}$ \\
\hline
\end{tabular}

TABLE III. MASS Moments of Inertia (ABout Pitch AXIS)

\begin{tabular}{|c|c|}
\hline $\begin{array}{c}\text { Mass Moment of Inertia of } \\
\text { Beam }\end{array}$ & $5.5 \times 10^{-5} \mathrm{kgm}^{2}$ \\
\hline $\begin{array}{c}\text { Mass Moment of Inertia of } \\
\text { Rotary Base }\end{array}$ & $1.6 \times 10^{-4} \mathrm{kgm}^{2}$ \\
\hline $\begin{array}{c}\text { Mass Moment of Inertia of } \\
\text { Wing }\end{array}$ & $6.6 \times 10^{-4} \mathrm{kgm}^{2}$ \\
\hline
\end{tabular}

The free rotary base that holds the beam is connected to translational springs on a pulley. The pitch stiffness, $K_{\theta}$, was found to be $0.24 \mathrm{Nm} / \mathrm{rad}$. 
Pitch data was measured using contactless potentiometers. The potentiometer used for the large amplitude oscillation (LAO) tests was the RLS RMA05A3A00 contactless rotary magnetic actuator, and the corresponding RM22VA0010B10F1B00 encoder body, (resolution of $0.5^{\circ}$ ). The potentiometer used for the small amplitude oscillation (SAO) tests utilized the Contelec Co-Vert-X 22E2 $836 \quad 221 \quad 505 \quad 78107 \quad 1307 \quad 1$ (resolution of $0.18^{\circ}$ ). LAO and SAO will be further discussed in section 3 .

The beam was outfitted with two PCB Piezoelectronics $352 \mathrm{C} 22$ accelerometers and eight Vishay CEA-13-240UZ-120 strain gauges (four for bending and four for torsion). The data from the accelerometers fed into a PCB 483C signal conditioner. The strain gauge data fed into a model 2120 Strain Gauge signal conditioner. The conditioned strain gauge and accelerometer data, along with the potentiometer data, fed into the National Instruments cDAQ 9174, which connected to the computer and LabVIEW via USB; see Fig. 2.

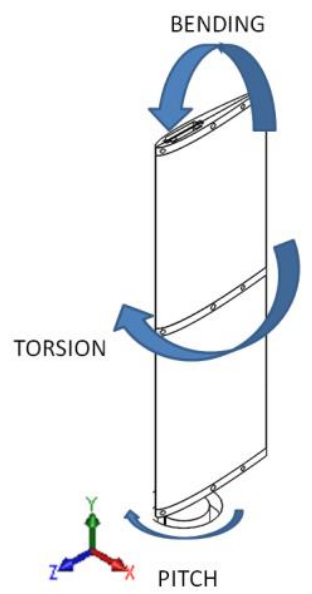

Figure 1. Illustration of Pitch, Bending and Torsion Motion

\section{Considerations for Design and Testing}

The wing was supported in the wind tunnel such that the wing support was flush with the wing tunnel floor. The ratio of wing span to tunnel height was calculated and found to be less than 0.6 , which ensured that the effects of the tunnel wall were negligible. [2].

The centre of gravity (CG) of the wing and beam combination was found to be $0.057 \mathrm{~m}$ aft of the leading edge (LE). Fig. 3 depicts the relative locations of the aerodynamic centre (AC), centre of gravity (CG), elastic axis (EA), and pitch axis (PA). In the current configuration, the EA, PA, and $\mathrm{AC}$ are all aligned.

The maximum blockage was calculated to be $8.3 \%$ (wing at $90^{\circ}$ pitch angle) while the minimum blockage was calculated to be $1.0 \%$ (wing at $0^{\circ}$ pitch angle).

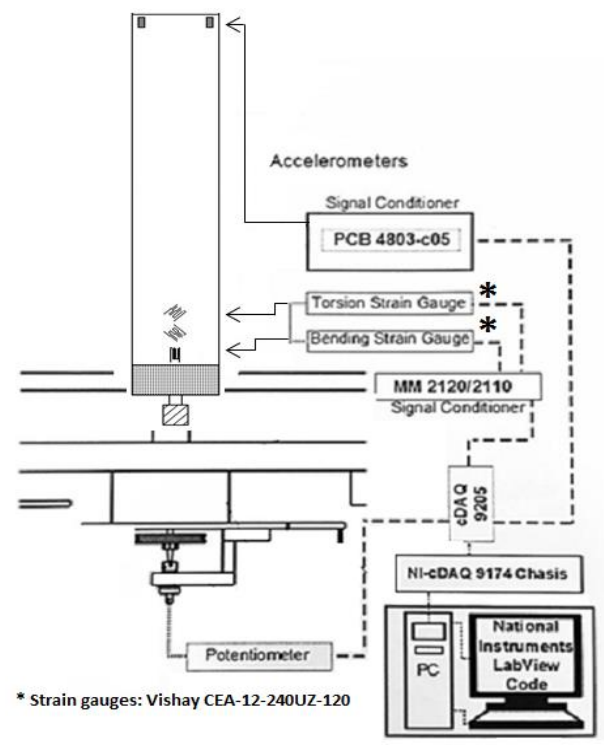

Figure 2. Experimental Test Set-Up

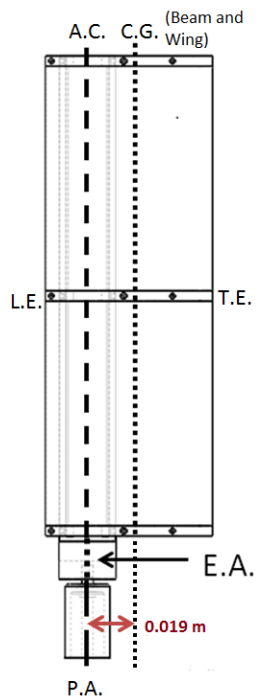

Figure 3. Relevant System Axes

\section{METHODOLOGY}

\section{A. Testing Procedure}

A total of six valid tests were performed. The test section was installed and lined up to ensure symmetry with the wind tunnel; the wing was set to zero angle of attack and set vertically straight.

The instrumentation was turned on to allow at least 20 minutes before testing, which allowed time for steady state to be reached. The wires were hooked up, isolated, and loosely taped to create support but still mitigate adding to the damping of the wing. In order to assess the ambient and equipment noise for each test, three recordings were done with the wind tunnel off, motor on, and motor and clutch on at zero RPM. 
The sampling frequency was $1000 \mathrm{~Hz}$. This data would be used two-fold: to find the baseline noise frequencies of the system and calculate the mean bias of the wing. Following this, the first type of ground vibrational testing (GVT1) was performed. GVT1 consisted of two free decay tests: one test with the pitch lock off (allowing for bending, torsion, and pitch, with a pitch initial condition) and one test with the pitch lock on (bending and pitch only, with a bending initial condition). With the pre-test GVT1 complete, tests were performed at various airspeeds, in combinations of increasing or decreasing airspeed sweeps, with the goal of obtaining the behavior of different amplitudes of LCO's. In an effort to locate the LCO branches, initial conditions were applied to the wing in the form of pitch perturbations and changes in airspeed. When changing sweep direction, hysteresis was noted in response due to the change in initial conditions via airspeed [3]. Following the airspeed tests, GVT1 (i.e. no-flow) was once again performed, wherein the purpose was to check if the system parameters changed. Following GVT1, the second type of ground vibrational was performed (GVT2), which was comprised of two free decay tests (with pitch initial conditions) for each of the three configurations, defined as such: S1 (rotary base, shaft, pulley, and encoder), S2 (rotary base, steel beam, eight strain gauges, two accelerometers, wires, shaft, pulley, encoder, and adhesives), and S4*(rotary base, steel beam, NACA 0012 wing, eight strain gauges, two accelerometers, wires, shaft, pulley, encoder, and adhesives) configurations as seen in Fig. 4-6, respectively. This data would allow us to find the decay rate and damping coefficient of the system in future work.

The $1^{\text {st }}$ and $2^{\text {nd }}$ analytical natural frequencies were calculated for bending and torsion of the S2 configuration (based on elastical beam theory), and the pitch damped natural frequency was experimentally determined for the S2 configuration as well, as seen in Table IV.

TABLE IV

NATURAL FREQUENCIES ( $\mathrm{S} 2$ CONFIGURATION)

\begin{tabular}{|l|l|l|}
\hline \multicolumn{3}{|l|}{ Natural Frequencies (Hz) based on [4], [5], [6], and [7] } \\
\hline Motion & Mode 1 & Mode 2 \\
\hline Pitch & 5.3 & n/a \\
\hline Bending & 6.0 & 38 \\
\hline Torsion & 102 & 308 \\
\hline
\end{tabular}

Note that the bending and torsion modes (as defined by the motion of the EA) are uncoupled due to the beam uniformity. The pitch motion is also considered to be uncoupled from the torsion motion due to the large difference in frequency scale. This assumption was confirmed from results of a finite difference (FD) solution of the $\mathrm{S} 2$ configuration. FD modeling of the $\mathrm{S} 4 *$ configuration resulted in the first three modes at $2.56 \mathrm{~Hz}, 5.0 \mathrm{~Hz}$ and $29.4 \mathrm{~Hz}$. These numerical results also match those obtained from the GVT. The first mode is pitch dominated, and the second mode is bending dominated.

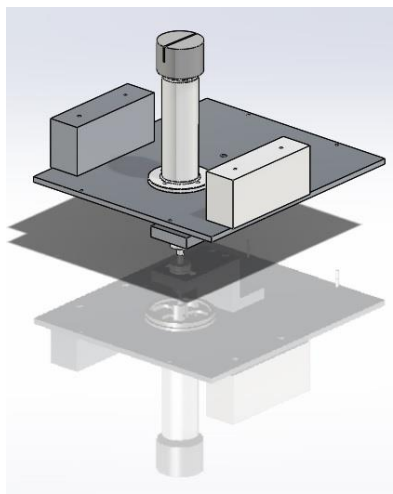

Figure 4. S1 Configuration (Rotary base only)

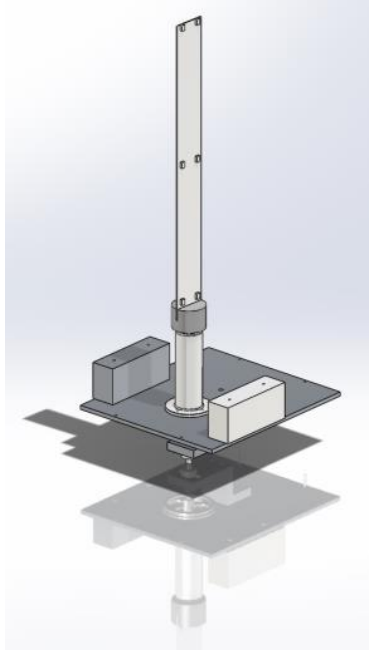

Figure 5. S2 Configuration (Rotary base and Beam)

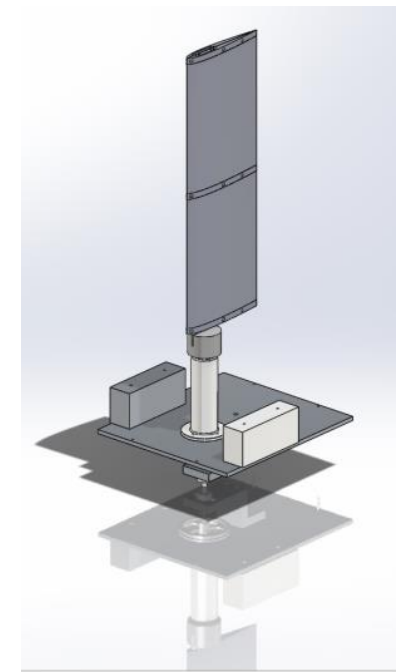

Figure 6. S4* Configuration (Rotary base, Beam and Wing) 


\section{B. Analysis Procedure}

The data obtained from testing was recorded into text-files using LabView, which were then manipulated into suitable excel files for post-processing with Matlab code. The aforementioned codes first took the potentiometer noise data, and converted it into root pitch angle (in degrees). The data was sorted in ascending order to find the fundamental frequency, which in turn was used to filter the data and find the mean bias of the filtered data portion which had a Gaussian distribution.

The ambient test data (temperature, pressure) were used to find the air density, airspeed, and Reynolds number. The varying airspeed data was then filtered and adjusted using a low pass filter, and the mean bias. The time histories were filtered to remove high frequency noise, since they have no impact on the dynamics. The time history served to visually indicate where the LCO behavior [3] occurred and select an appropriate portion of the signal, as well as to confirm if steady-state had been attained. If no global trends indicating increasing/decreasing root pitch angle was observed, the data was assumed to be gleaned during steady state. Histograms served to provide information on the data spread and whether the dynamics were symmetrical, along with the even harmonics of the PSD's which further confirmed symmetry. Once the data was deemed properly selected and filtered, the mean and mode positive pitch angle peak amplitudes were located using the filtered time history data, while the LCO frequency was found using the filtered root pitch angle PSD's. This was performed for all five tests and yielded final values of mean and mode positive root pitch angle peak amplitudes, pitch LCO frequency, airspeed, and Reynolds number.

\section{RESULTS AND DISCUSSION}

\section{A. Positive Mean Peak Amplitude of Root Pitch Angle Results}

Values of the mean positive peak amplitude of the root pitch angle were plotted versus airspeed for all five tests (Fig. 7). The mode positive peak amplitude of the root pitch angle values were also found, and determined to be sufficiently close to the mean values, thus further substantiating that the time response was symmetrical.

It is worthy to confirm that the range of airspeeds corresponded to a Reynolds number range of $9.11 \times 10^{4}<\operatorname{Re}_{c}$ $<1.19 \times 10^{5}$, which is indeed in the range of transitional Reynolds number. Previous research has observed that the range of Reynolds numbers where LCO's occur is $5.5 \times 10^{4}<$ $\mathrm{Re}_{\mathrm{c}}<1.2 \times 10^{5}[8]$. Hitherto for the quasi-2D rigid wing, elastically mounted in pitch and heave, two LCO regions (also called branches) had been observed [8]. These regions can be described as SAO and LAO. In this paper, we tentatively define the ranges of $0^{\circ}-15^{\circ}$, and $30^{\circ}-70^{\circ}$ for SAO and LAO, respectively.
Referring to Fig. 7, it is substantial to notice that along with the evident SAO and LAO branches, a third branch is observed, which we have deemed very large oscillations (VLAO) and tentatively defined as the range of $70^{\circ}-120^{\circ}$.

It is important to note that the LAO region across all tests did not appear to be stationary and at steady state, i.e. there was significant amplitude modulation and a slight global increase in peak amplitude over time. No explanations are offered at this time.

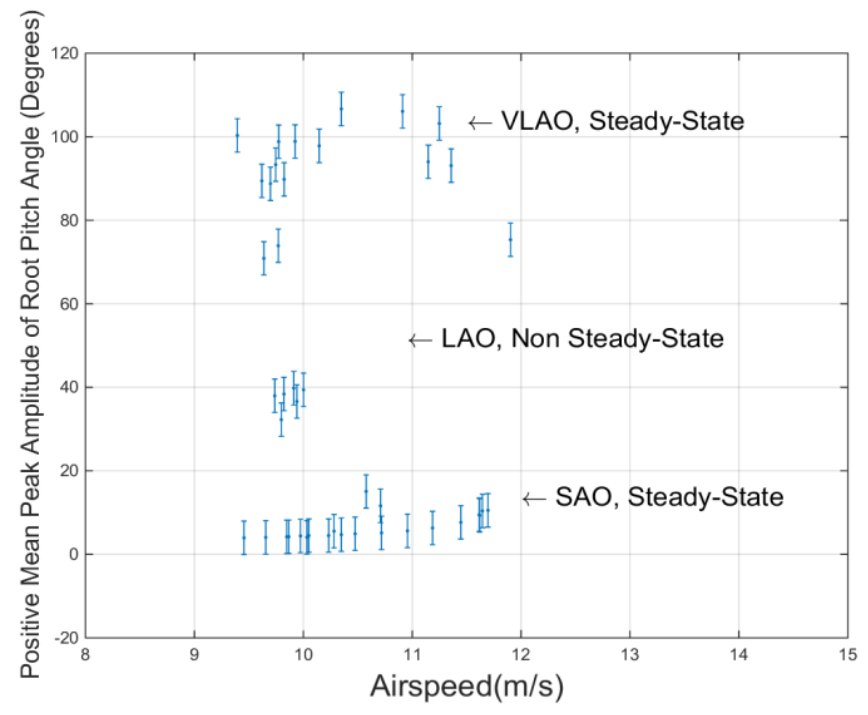

Figure 7. Positive Mean Peak Amplitude of Root Pitch Angle Versus Airspeed

It is meaningful to state that the wing could possibly be experiencing coalescence flutter or stall flutter for the LAO and VLAO branches. For the possibility of coalescence flutter, this could be a result of the coupling of the pitch and $1^{\text {st }}$ bending mode as these two frequencies are close to each other (Table IV); ergo, data reduction of the torsion and bending information is an important part of future work ponderations. The information can then be compared to the bending and torsion natural frequencies in Table IV.

This preliminary focus on pitch data is important because we use it as a guide; if we ascertain that it is well behaved, we thusly assume that the bending and torsion data will also be well behaved.

While we theorize that SAO is caused by laminar flow separation, along with the free pitching wing motion which causes negative aerodynamic damping, the physical mechanisms which cause LAO and VLAO are still to be confirmed. Hence, LAO and VLAO will be the primary focus, with the SAO serving to confirm that the Reynolds number effect is present, which provides information on the state of the flow. For our testing, the existence of the Reynolds number effect confirms that the flow is laminar and the wing is smooth. 


\section{B. LCO Frequency Results}

The LCO frequencies as measured from the root pitch angle were also found, and shown in Fig. 8. For the LCO frequency behaviour (Fig. 8), an average frequency resolution $f=1 / F_{\mathrm{s}} \Delta t$ $=0.0305 \mathrm{~Hz}$ was used, since $\Delta t$ varied for each test. From Fig. 8 , we see that once again, there are three distinct branches, corresponding to SAO, LAO and VLAO. All LCO frequencies are within the small frequency spectrum of $2.5-4.5 \mathrm{~Hz}$. Note that these are lower than the experimental natural pitch frequency of approximately $5 \mathrm{~Hz}$. Overall, as the mean peak amplitude of the root pitch angle increases, the LCO frequency decreases. Also for consideration is that the work from the quasi-2D wing [8] observed that the SAO had an approximate frequency range of $2.5-5 \mathrm{~Hz}$, whereas $\mathrm{LAO}$ had an approximate frequency range of $2-2.5 \mathrm{~Hz}$ (for a frequency ratio of 0.74). This is parallel to the results shown in this paper, where the frequency ranges are approximately $3.7-4.5$ $\mathrm{Hz}$ for SAO, $3.2-3.7 \mathrm{~Hz}$ for LAO and $2.8-3.1 \mathrm{~Hz}$ for VLAO.

While this work provides a good basis in the ongoing research to characterize the dynamics of a free pitching flexible cantilever NACA0012 wing, more detailed work is required. For instance, FEA modelling will be performed, where we will attempt to couple finite elements with a simple aerodynamic model, using Theodorsen's or Wagner's functions for instance for the linear case, as well as performing a modal analysis. Additionally, the strain gauge and accelerometer data will be fully analyzed. LAO tests will be redone in order to try and attain steady-state results. Post-work calculations will also include assessing the damping and stiffness values.

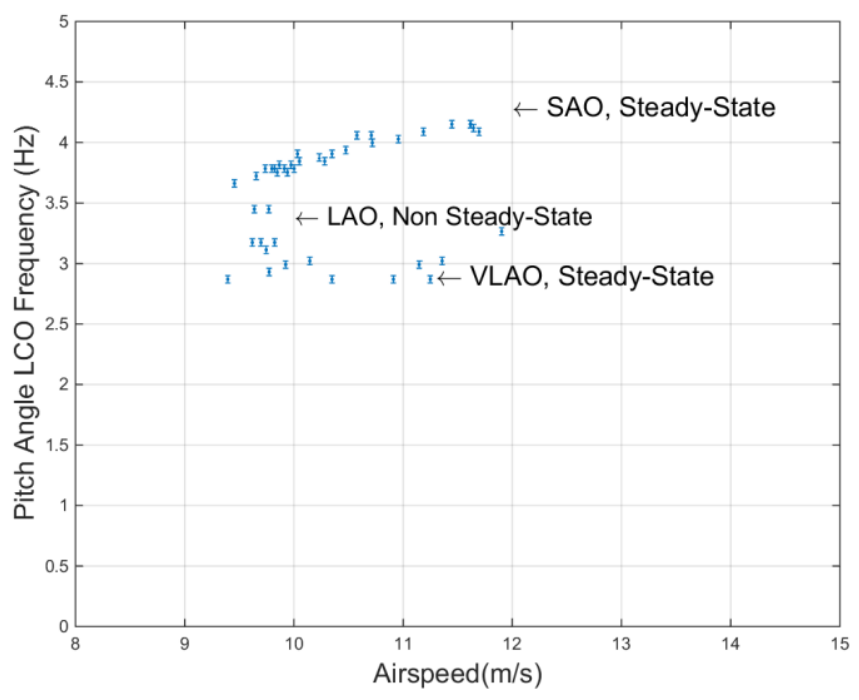

Figure 8. Pitch Angle LCO Frequency Versus Airspeed

\section{CONCLUSIONS}

A series of tests were performed on a free pitching flexible cantilever NACA0012 wing, in order to characterize the dynamics at transitional Reynolds numbers. Data was recorded using a potentiometer, strain gauges (in bending and torsion) and accelerometers. The potentiometer data was filtered and used to generate plots of the mean positive peak amplitude of the root pitch angle versus airspeed and LCO frequency versus airspeed. Three LCO branches were found corresponding to SAO, LAO and VLAO. It is theorized that the SAO is caused by flow separation at small angles of attack due to transitional Reynolds number and wing free pitching, which in turn causes negative aerodynamic damping. The mechanisms responsible for LAO and VLAO have yet to be confirmed through future testing and analysis. The range of Reynolds numbers where LCO's are observed, as well as the LCO frequencies are consistent with previously published work suggesting either coupled or stall flutter in these cases. Further tests will be performed to better capture LAO. Furthermore, the strain gauge and accelerometer data will be analyzed. FEA and numerical modelling will be done and used for corroboration of results, and post-work damping and stiffness values will be calculated.

\section{ACKNOWLEDGMENTS}

The authors gratefully acknowledge the help and support of Dr. Azemi Benaissa, M. Lavoie, Jeff Fleming, Brendan Freeman, Charles Sadiq, Pierre Seguin, Pierre Bissonnette, and David Babcock, and the other members of the Design Laboratory.

\section{REFERENCES}

[1] D. Poirel, Y. Harris, and A. Benaissa. Self-sustained aeroelastic oscillations of a NACA 0012 airfoil at low-to-moderate Reynolds numbers. Journal of Fluids and Structures, Vol. 24, No.5, pp. 700-719, 2008.

[2] J.B Barlow, W.H. Rae, Jr., A. Pope. Low-speed wind tunnel testing. John Wiley and Sons Inc., Toronto, Ontario. Third Edition, pp. 28, Feb. 221999.

[3] E.H. Dowell, J.Edwards, T.Strganac. Nonlinear aeroelasticity. Journal of Aircraft Vol.40, No.5, pp.857-874, September-October 2003.

[4] A. Bedford and W. Fowler. Engineering mechanics statics and dynamics. Prentice Hall, Upper Saddle River, New Jersey 07458, 2002

[5] D.H Hodges, and G. Alvin Pierce. Introduction to structural dynamics and aeroelasticity. Cambridge university press 40 west 20th street, New York, NY. 10011-11-4211, USA, pp. 35-36, 2002

[6] P.P. Benham, R.J. Crawford, and C.G. Armstrong. Mechanics of engineering materials. Pearson Education Limited, Longman Group Limited (C, pp.115-116, 1996.

[7] W.H. Thomson. Theory of vibration with applications. Prentice Hall Inc. Englewood Cliffs N.J., pp. 273, 1972.

[8] D. Poirel and F. Mendes. Experimental small amplitude self-sustained pitch-heave oscillation at transitional Reynolds numbers. AIAA Journal Vol.52 No.8, pp.1581-1590, August 2014 